Pacific Journal of Mathematic 


\title{
FINITE GROUPS WITH CHEVALLEY-TYPE COMPONENTS
}

\author{
N. BuRgoyne
}

\begin{abstract}
This article contains the proof of one part of the unbalanced group conjecture of Aschbacher, Thompson and Walter.
\end{abstract}

1. Introduction. In [13] Thompson discussed simple groups $X$ such that $O\left(C_{X}(\alpha)\right) \neq 1$ for some involution $\alpha \in$ Aut $X$ and sketched a proof of the theorem stated below in $\S 3$. The key to this proof is a recent result of Aschbacher [2]. Some detailed properties of Chevalley type groups over finite fields of odd characteristic are also required.

The purpose of this article is to prove the necessary properties of Chevalley type groups (see $\S \S 5$ and 6 ). To motivate these results it seemed worthwhile to review the arguments in [13]. This occupies $\S \S 3$ and 4.

2. Notation. Let $X$ denote a finite group, then

$$
\begin{aligned}
& \text { Inv } X=\left\{\alpha \in X: \alpha^{2}=1, \alpha \neq 1\right\} \\
& \text { Comp } X=\left\{Y: Y \triangleleft \triangleleft X, Y=Y^{\prime}, Y / 0(Y) \text { quasi-simple }\right\} \\
& L(X)=\text { product of all } Y \in \operatorname{Comp} X .
\end{aligned}
$$

For properties of $L$, in particular $L$-balance and its implications, see [5].

$$
\begin{aligned}
\mathscr{E} & =\left\{X: L(X) \text { simple, } C_{X}(L(X))=1\right\} \\
\mathscr{C} & =\left\{X \in \mathscr{E}: O\left(C_{X}(\alpha)\right) \neq 1 \text { some } \alpha \in \operatorname{Inv} X\right\} .
\end{aligned}
$$

For convenience, the known groups in $\mathscr{C}$ are divided into four disjoint families.

$$
\begin{aligned}
& \mathscr{C}_{1}=\left\{X \in \mathscr{C}: L(X) \simeq L_{2}(q) \text { or } A_{7}, q=\text { odd }\right\} \\
& \mathscr{C}_{2}=\left\{X \in \mathscr{C}: L(X) \simeq L_{3}(4) \text { or Held's group [9] }\right\} \\
& \mathscr{C}_{3}=\left\{X \in \mathscr{C}: L(X) \simeq A_{n}, n \geqq 9 \text { and odd }\right\} \\
& \mathscr{C}_{4}=\{X \in \mathscr{C}: L(X) \text { a Chevalley type group of } \\
& \text { odd characteristic, but not an } \left.L_{2}(q)\right\} .
\end{aligned}
$$

In general, our notation follows Gorenstein [7].

3. The grand conjecture. This states that $\mathscr{C}=\mathscr{C}_{1} \cup \mathscr{C}_{2} \cup$ $\mathscr{C}_{3} \cup \mathscr{C}_{4}$. Thompson's attack on this conjecture starts with the following proposition. Its proof depends on several long and difficult results. 
Proposition. Let $G \in \mathscr{C l}$ and assume $|G|$ minimal subject to $G \notin \mathscr{L}_{1} \cup \mathscr{L}_{2} \cup \mathscr{L}_{3} \cup \mathscr{H}_{4}$. For $\alpha \in \operatorname{Inv} G$ let $\Gamma_{\alpha}=\left\{\beta \in \operatorname{Inv} C_{G}(\alpha)\right.$ : $\left.O\left(C_{G}(\alpha)\right) \cap C_{G}(\beta) \nsubseteq O\left(C_{G}(\beta)\right)\right\}$. Then,

(i) $\Gamma_{\alpha}$ is nonempty for some $\alpha \in \operatorname{Inv} G$.

(ii) If $\beta \in \Gamma_{\alpha}$ and $D=O\left(C_{G}(\alpha)\right) \cap C_{G}(\beta)$ then there exists a $Y \in$ Comp $C_{G}(\beta)$ normalized by $D$ and such that $[D, Y / O(Y)] \neq 1$.

Proof. (i) If $S C N_{3}(2)$ of $G$ is empty then by [11] $G$ has sectional 2-rank at most 4 . Hence by [8] $G$ is a known group. Thus if (i) is false, since $S C N_{3}(2)$ is nonempty, the results of [4], [6], [1] may be used. They imply that $G$ is known.

(ii) An extended form of $L$-balance, see [5], implies that $D$ normalizes each element of Comp $C_{G}(\beta)$. Since $D \nsubseteq O\left(C_{G}(\beta)\right)$ the result follows from elementary properties of $L$.

Let $G, \alpha, \beta, D, Y$ be defined as in the proposition. Then a lemma in [6] gives $Y^{\alpha}=Y$ and $[\alpha, Y / O(Y)] \neq 1$. Put $M=\langle\alpha, D, Y\rangle$ and $M^{*}=M / Z^{*}(M)$ then $M^{*} \in \mathscr{C}$ and so, by the choice of $G, M^{*} \epsilon$ $\mathscr{K}_{i}$ for some $i \in\{1,2,3,4\}$.

THEOREM. $\quad M^{*} \notin \mathscr{M}_{4}$.

The proof will be given in the following sections. The result of [2] will be used in the following form.

AschbaCher's THEOREm. Let $X \in \mathscr{M}, \gamma \in \operatorname{Inv} X, L \in \operatorname{Comp} C_{X}(\gamma)$. Suppose $L$ has 2-rank equal to 1 and $\gamma \in L$ then $X \in \mathscr{M}_{4}$.

The grand conjecture directly implies the $B$-conjecture, namely; $B(G) \supseteqq B\left(N_{G}(T)\right)$ for any finite group $G$ and $T$ any 2-subgroup of $G$, where $B(X)=$ product of all $Y \in$ Comp $X$ with $Y$ not quasisimple.

4. Proof of the theorem. Let $G, \alpha, \beta, D, M, M^{*}$ be defined as in $\S 3$ and assume $M^{*} \in \mathscr{K}_{4}$. Using Aschbacher's theorem and the results proved in $\S 5$ and $\S 6$ we proceed, as in [13], to obtain a contradiction.

Let $E \subseteq D$ and let $A / B$ be some section of $G$ : we say that $\langle\alpha, E\rangle$ 'acts properly' on $A / B$ if $\langle\alpha, E\rangle$. normalizes $A$ and $B$ and $C_{\langle\alpha, E\rangle}(A / B)$ is a proper subgroup of $E$ (possibly 1 ). Thus $E \neq 1$ and, to begin, we know that $\langle\alpha, D\rangle$ acts properly on $M^{*}$.

Step 1. By Proposition A of $\S 5$ (with $X=M^{*}, t=\alpha$ and $Y=E$ ) there exists $\gamma^{*} \in \operatorname{Inv} M^{*}$ and $S^{*} \in \operatorname{Comp} C_{M}^{*}\left(\gamma^{*}\right)$ with $S^{*} \simeq S L_{2}(q)$ for 
some odd $q,\left\langle\gamma^{*}\right\rangle=Z\left(S^{*}\right)$, and $\langle\alpha, D\rangle$ acts properly on $S^{*}$.

Choose $S_{1}$ to be the full inverse image of $S^{*}$ in $M$ and put $S_{2}=S_{1}^{(\infty)}$. Choose $\gamma \in \operatorname{Inv} Z^{*}\left(S_{2}\right)$ so that $[\gamma, \alpha]=1$ and put $S=$ $C_{S_{2}}(\gamma)^{(\infty)}$. By construction, $\gamma \in C_{G}(\langle\alpha, \beta\rangle)$ and so $\gamma$ normalizes $D$. Put $D_{1}=C_{D}(\gamma)$ then, since $[D, \gamma] \subseteq D \cap O(S) \neq D$, we see that $\left\langle\alpha, D_{1}\right\rangle$ acts properly on $S / O(S)$.

Since $S \triangleleft \triangleleft C_{M}(\gamma)$ therefore $S \in \operatorname{Comp} C_{G}(\langle\beta, \gamma\rangle)$. Let $K$ be the normal closure of $S$ in $L\left(C_{G}(\gamma)\right)$. Then by $L$-balance either,

(a) $K \in \operatorname{Comp} C_{G}(\gamma)$ and $K^{\beta}=K$, or

(b) $K=K_{1} K_{2}$ with $K_{1}, K_{2} \in \operatorname{Comp} C_{G}(\gamma), K_{1}^{\beta}=K_{2}$ and $K / O(K) \simeq$ $S L_{2}(q) \times S L_{2}(q)$.

Furthermore, $\left\langle\alpha, D_{1}\right\rangle$ acts properly on $K / O(K)$ and, in case (b), on each $K_{i} / O\left(K_{i}\right)$. In case (a), since $\gamma \in K, K / O(K)$ is a nontrivial covering of $K / Z^{*}(K)$.

In the next two steps we will show that cases (a), (b) both lead to the following configuration:

$W \simeq Z_{2} \times Z_{2}$ is a subgroup of $G$ with $N_{1}, N_{2} \in \operatorname{Comp} C_{G}(W)$ (*) such that, if $N=N_{1} N_{2}$, then $N / O(N) \simeq S L_{2}(q) \times S L_{2}(q)$ for some odd $q, W \subseteq Z(N)$, and $\langle\alpha, E\rangle$ acts properly on each $N_{i} / O\left(N_{i}\right)$ for some $E \subseteq D$.

Step 2. In case (a) put $J=\left\langle\alpha, \beta, D_{1}, K\right\rangle$ and $J^{*}=J / Z^{*}(J)$. Then $J^{*} \in \mathscr{C l}$ and so $J^{*} \in \mathscr{C}_{i}$ for some $i \in\{1,2,3,4\}$. If $J^{*} \in \mathscr{C}_{1}$ then Aschbacher's theorem (with $X=G, L=K$ ) contradicts our choice of $G$. If $J^{*} \in \mathscr{C}_{2}$ then, since Held's group has no proper covering, $L\left(J^{*}\right) \simeq L_{3}(4)$ and the calculation in $\S 6$ yields a contradiction. If $J^{*} \in \mathscr{C l}_{3}$ the results in [12] contradict the choice of $G$. Hence $J^{*} \in$ $\mathscr{M}_{4}$. In this case we may use Proposition B of $\S 5$ (with $X=J^{*}$, $t=\alpha, s=\beta$ and $\hat{L}=K O(J) / O(J))$. This gives configuration (*) in $K O(J) / O(J)$ and arguing as in the second paragraph of Step 1 we see that $(*)$ also occurs in $G$.

Step 3. In case (b), since $\gamma \in Z(K)$, we may choose $\rho \in \operatorname{Inv} Z^{*}(K)$ with $\rho \neq \gamma$ and so that $\rho$ normalizes $\left\langle\alpha, D_{1}\right\rangle$. Hence $[\rho, \alpha]=1$ and putting $D_{2}=C_{D_{1}}(\rho)$ we see, as in Step 1, that $\left\langle\alpha, D_{2}\right\rangle$ acts properly on each $K_{i} / O\left(K_{i}\right)$. Then $W=\langle\gamma, \rho\rangle$ and $N=C_{K}(W)^{(\infty)}$ give the configuration $\left({ }^{*}\right)$.

Step 4. We may assume (*). Put $\left\langle\delta_{i}\right\rangle=N_{i} \cap W$, so that $W=$ $\left\langle\delta_{1}, \delta_{2}\right\rangle$, and put $C=G_{G}\left(\delta_{1}\right)$. Then, by $L$-balance, we have $N \subseteq L(C)$. Hence $W \subseteq L(C)$ and so $\delta_{2}$ normalizes each element of Comp $C$. Thus if $H_{i}$ is the normal closure of $N_{i}$ in $L(C)$, then $H_{i} \in$ Comp $C$.

Suppose $\left[H_{1}, \delta_{2}\right] \subseteq O\left(H_{1}\right)$. Then $H_{1}=O\left(H_{1}\right) C_{H_{1}}\left(\delta_{2}\right)$ and, since 
$N_{1} \triangleleft \triangleleft C_{H_{1}}\left(\delta_{2}\right)$, we have $H_{1}=O\left(H_{1}\right) N_{1}$, By Aschbacher's theorem this contradicts our choice of $G$. Thus $\left[H_{1}, \delta_{2}\right] \mp O\left(H_{1}\right)$ and, since $\delta_{2} \in H_{2}$ we must have $H_{1}=H_{2}$.

Put $H .=\left\langle H_{1}, \alpha, E\right\rangle$ and $H^{*}=H / Z^{*}(H)$ and put $\delta_{2}^{*}, N_{\imath}^{*}$ for the images of $\delta_{2}, N_{i}$ in $H^{*}$. Then $N_{i}^{*} \in \operatorname{Comp} C_{H}^{*}\left(\delta_{2}^{*}\right)$ and $N_{1}^{*} \simeq L_{2}(q)$ while $\left\langle\delta_{2}^{*}\right\rangle \in N_{2}^{*} \simeq S L_{2}(q)$. By Aschbacher's theorem $H^{*} \in \mathscr{C}_{4}$ and so Proposition $\mathrm{C}$ of $\S 5$ applies (with $X=H^{*}, R_{i}=N_{i}^{*}$, and $r=\delta$ ). We have $H^{*} \simeq B_{3}(q)$ and, since $\langle\alpha, E\rangle$ acts properly on each $N_{\imath}^{*}$, a contradiction.

Hence $M^{*} \notin . \mathbb{K}_{4}$.

This last step, the reduction to the seven dimensional orthogonal group $B_{3}(q)$, is at the heart of the argument. This point is made in the closely related work of Walter [14].

5. Results on Chevalley-type groups. We now apply the methods of [3] to prove Propositions A, B, C. Together with the arguments in $\S 6$, this will complete the proof that $M^{*} \notin \mathscr{C}_{4}$. At several points the proofs of the propositions reduce to case by case calculations. These are always straightforward applications of the theory in [3] and are therefore omitted.

The notation of [3] is followed closely: thus $G$ will now denote a connected, simple algebraic group over an algebraically closed field k. $T$ is a maximal torus of $G$ and $X(T), \Gamma(T)$ are the associated lattices. $\Sigma$ is the root system in $X(T)$ and $W=N_{G}(T) / T$ the Weyl group. We assume that rank $G=r$ is $\geqq 2$ and that the characteristic of $k$ is $p$, an odd prime. Since $G$ is simple we may take $X(T)$ to be the adjoint lattice, i.e., spanned by $\Sigma$. Let $\Pi=$ $\left\{\alpha_{1}, \cdots, \alpha_{r}\right\}$ be a simple root system in $\Sigma$ and $\left\{\eta_{1}, \cdots, \eta_{r}\right\}$ the dual basis in $\Gamma(T)$. Let $\alpha_{*}=-\left(m_{1} \alpha_{1}+\cdots+m_{r} \alpha_{r}\right)$ be the low root in $\Sigma$ relative to $\Pi$ and $\hat{\alpha}_{*} \in \Gamma(T)$ its co-root.

To avoid confusion with the above notation, the involutions $\alpha, \beta, \gamma, \delta, \cdots$ occurring in $\S \S 1-4$ are replaced by lower case latin letters. Since the calculations of this section are completely independent of the earlier sections this should not cause any trouble. Note that if $H$ is some connected reductive algebraic group then $E(H)$ is used to denote its maximal semi-simple subgroup and $F(H)$ to denote the largest central torus of $H$. Thus $[E(H), F(H)]=1$ and $E(H) F(H)=H$ (see [3] $\S 2$ ). Context should enable one to avoid confusion with the corresponding symbols in finite group theory.

In the following table we list (1) the simple Chevalley groups and their extended Dynkin diagrams. Each simple root is numbered and $\alpha_{*}$ is denoted by $*,(2)$ a representative in $T$ for each class of 
involutions in the group. Here " $\eta_{i}$ " is short for $\eta_{i}(-1) \in T$, (3) the (quasi) simple components of the centralizer of each involution. Certain obvious conventions are used: $A_{0}=B_{0}=C_{0}=1, A_{1}=B_{1}=C_{1}$, $B_{2}=C_{2}, A_{3}=D_{3}$, and $D_{1}$ is not simple and should be omitted while $D_{2}$ has two components, each of type $A_{1}$.

Similar results for the graph automorphisms are tabulated in $\S 4.3$ of [3].

The methods of [3] are to a certain extent based on the earlier work of Iwahori [10]. This useful paper contains several very detailed computations of classes and centralizers of involutions.

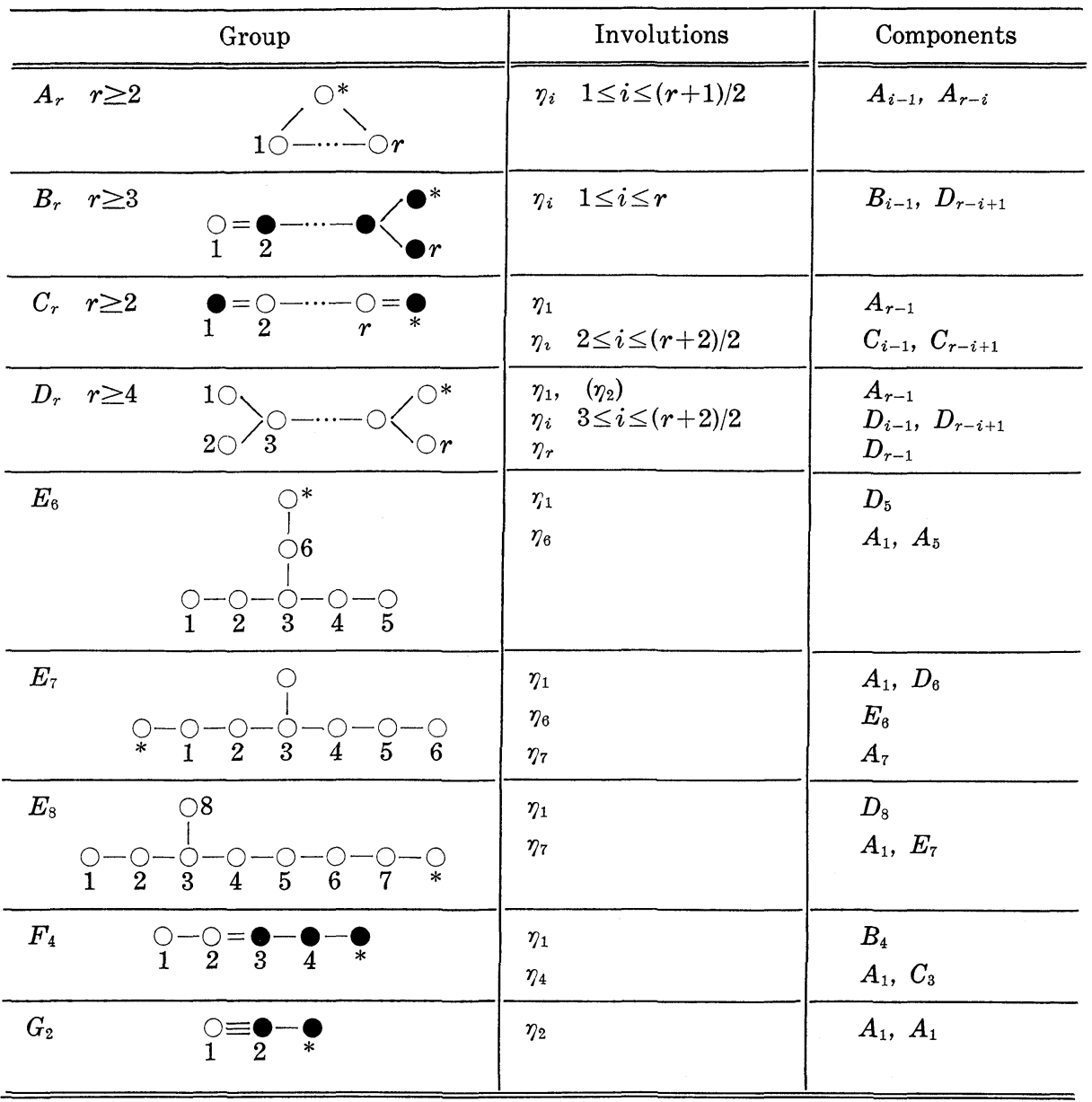

O = long root. In $D_{r}, \eta_{1}$ and $\eta_{2}$ are (are not) conjugate if $r$ is odd (even).

The finite groups corresponding to $G$ are the fixed point sets $G(\rho)=\{g \in G: \rho g=g\}$ where $\rho$ is a finite type endomorphism of $G$ ( $\$ 5.1$ in [3]). We may assume that $\rho$ stabilizes $T$ and hence $\rho=$ $i_{n} \sigma$, where $i_{n} g=n g n^{-1}$ with $n \in N_{G}(T)$, and $\sigma$ is in standard form 
relative to $T$ and $\Pi$, i.e.,

$$
\sigma x_{ \pm \alpha}(\xi)=x_{ \pm \alpha \alpha}\left(\xi^{q \alpha}\right) \quad \text { all } \quad \alpha \in \Pi, \quad \xi \in k
$$

where $\alpha \rightarrow \sigma \alpha$ is a permutation of $\Pi$ and $q_{\alpha}$ is a power of $p$. The distinct pairs $G, \sigma$ produce all possible finite Chevalley type groups $G(\sigma)\left(\right.$ and $G(\sigma) \simeq G(\rho)$ when $\left.\rho=i_{n} \sigma\right)$.

By the lemma below, $G$ of type $G_{2}$ will not occur, and hence, since $r \geqq 2$ and $p=$ odd, we have $L(G(\sigma))=O^{p^{\prime}}(G(\sigma))$. Thus there is a natural embedding $L(G(\sigma)) \leqq G(\sigma) \subseteq$ Aut $L(G(\sigma))$ with $G(\sigma)$ consisting of all the inner and diagonal automorphisms. The usual notation, e.g., $A_{2}(q), B_{3}(q),{ }^{2} E_{6}(q), \cdots$ is used to denote the simple groups $L(G(\sigma))$. In all cases which occur below, $q_{\alpha}=q$ for all $\alpha \in \Pi$.

If $w \in W$ is the image of $n \in N_{G}(T)$ then $\rho=i_{n} \sigma$ induces the action $\rho=w \sigma$ on $\Gamma(T)$ ( $\S 2.3$ in [3]). Since most calculations take place in $\Gamma(T)$ we usually describe $\rho$ in this latter form.

Let $X \in \mathscr{K}_{4}$ then we may find $G, \rho$ satisfying $L(X)=L(G(\rho))$.

Lemma. Let $X \in \mathscr{C}_{4}, t \in \operatorname{Inv} X$ and $Y=O\left(C_{X}(t)\right) \neq 1$, then

(a) $\langle L(X), t, Y\rangle \subseteq G(\rho)$

(b) $L(X)$ is not one of ${ }^{3} D_{4}(q), E_{8}(q), F_{4}(q), G_{2}(q),{ }^{2} G_{2}(q)$

(c) $|Y|$ divides $q-1$ or $q+1$ (in particular $q \neq 3$ ).

Proof. If $t$ induces a field or graph automorphism then $O\left(C_{X}(t)\right)=$ 1 as follow from $\S \S 5.5$. and 4.3 in [3]. Hence $t \in G(\rho)$. Since [ $Y$, $\left.L\left(C_{X}(t)\right)\right]=1$ no element in $Y$ can induce field automorphisms or (in the case of $D_{4}$ ) graph automorphisms and hence $Y \subseteq G(\rho)$. This proves (a).

Using the classification in $\S 3$ [3], with $\psi=1$ (see the above table), we may assume $t=\eta_{i}(-1)$ for some $1 \leqq i \leqq r$. If $C_{G}(t)$ is semi-simple then, since $X(T)$ is adjoint, $Z\left(C_{G}(t)\right)$ turns out to be a 2-group. This follows from inspection of the table (the only case that needs further calculation is the involution $\eta_{6}(-1)$ in $\left.E_{6}\right)$. Hence $F\left(C_{G}(t)\right) \neq 1$ (§2.1 in [3]) which implies that $m_{i}=1$ in the expansion of $\alpha_{*}$. This is immediate from the description of the centralizer subgroups as given in $\S 4$ of [3], see also Proposition 8 of [10]. This eliminates groups of type $E_{8}, F_{4}, G_{2}$. If $L(G(\rho))={ }^{3} D_{4}(q)$ then $\rho$ permutes cyclically the three involutions with $m_{i}=1$ and hence no conjugates of them can lie in $G(\rho)$. This proves (b).

Since $t=\eta_{i}(-1)$ and $m_{i}=1, F\left(C_{G}(t)\right)=\left\langle\eta_{i}(\zeta): \zeta \in k^{*}\right\rangle$, and so $Y \subseteq\left\langle\eta_{i}(\zeta):(\rho-1) \eta_{i}(\zeta)=1, \zeta \in k^{*}\right\rangle$. Since $\rho \eta_{i}= \pm q \eta_{i}$, this proves (c).

Proposition A. Let $X \in \mathscr{A}_{4}, \quad t \in \operatorname{Inv} X$ and $1 \neq Y \subseteq O\left(C_{X}(t)\right)$ 
then there exists $u \in \operatorname{Inv} X$ such that $S \in \operatorname{Comp} C_{X}(u)$ where $S \simeq$ $S L_{2}(q),\langle u\rangle=Z(S)$, and $\langle t, Y\rangle$ acts properly on $S$.

Proof. By the lemma we may suppose $X=G(\rho)$. We choose $u=\hat{\alpha}_{*}(-1)$ and put $S_{*}=\left\langle U_{\alpha_{*}}, U_{-\alpha_{*}}\right\rangle\left(\S 2.1\right.$ [3]). Since $\hat{\alpha}_{*}(-1) \in S_{*}$, we have $S_{*} \simeq S L_{2}(k)$. By inspection of the extended Dynkin diagram of $G$ we see that $S_{*}$ is always a factor of $E\left(C_{G}(u)\right)$. Now $\eta_{i}(\zeta) x_{\alpha_{*}}(\xi) \eta_{i}\left(\zeta^{-1}\right)=x_{\alpha_{*}}\left(\xi \zeta^{-1}\right)$ and hence $\langle t, Y\rangle$ acts properly on $S_{*}$. Thus it remains to show that $\rho$ may always be chose to stabilize $S_{*}$, for then $\langle t, Y\rangle$ will act properly on $S=S_{*}(\rho)$.

Let $v_{i} \in W$ be the unique element stabilizing the set $\left\{\alpha_{1}, \cdots, \alpha_{r}\right.$, $\left.\alpha_{*}\right\}$ and such that $v_{i} \alpha_{*}=\alpha_{i}$. Let $w_{0} \in W$ be the unique element such that $w_{0} \Pi=-\Pi$, and let $w_{0}^{(i)}$ be the corresponding element for the simple root system $\Pi-\left\{\alpha_{i}\right\}$. A simple argument yields $v_{i}=$ $w_{0}^{(i)} w_{0}$.

Let $\sigma$ be in standard form relative to $T$ and $\Pi$. The methods of $\S 5.3$ in [3] show directly that all possible pairs $(X, t)$ occur among $\left(G(\rho), \eta_{i}(-1)\right)$ where $m_{i}=1$ and $\rho=\sigma$ or $\rho=v_{i} \sigma$. When $\rho=\sigma$ it is clear that $\sigma$ stabilizes $S_{*}$ and we are done. However if $\rho=v_{i} \sigma$, then $\rho S_{*} \neq S_{*}$. In this case put $\rho^{\prime}=w_{0} \sigma$. Let $n_{0}^{(i)} \in N_{G}(T)$ be any inverse image of $w_{0}^{(i)}$. By the definition of $w_{0}^{(i)}$ we see, by $\S 4.2$ in [3], that $n_{0}^{(i)}$ lies in the connected component of $C_{G}(t)$. Hence, since $w_{0}=w_{0}^{(i)-1} v_{i}$ and $G\left(\rho^{\prime}\right) \simeq G(\rho)$, all pairs $(X, t)$ occur (up to isomorphism) among the pairs $\left(G\left(\rho^{\prime}\right), \eta_{i}(-1)\right)$ with $m_{i}=1$ and $\rho^{\prime}=\sigma$ or $\rho^{\prime}=w_{0} \sigma$. Since $w_{0} \alpha_{*}=-\alpha_{*}, \rho^{\prime}$ stabilizes $S_{*}$ and we are done.

Let $q_{1}$ be some power of $q$. In fact it will turn out that $q_{1} \in$ $\left\{q, q^{2}\right\}$.

Proposition B. Let $X \in \mathscr{C}_{4}$ and let $\hat{L}$ be a 2-fold covering of $L(X)$ and let $t, s \in \operatorname{Inv}($ Aut $\hat{L})$ such that

(i ) $Y=O\left(C_{\hat{L}}(t)\right) \neq 1$ and $[s,\langle t, Y\rangle]=1$,

(ii) there exists $\hat{Q} \in \operatorname{Comp} C_{\hat{L}}(s)$ such that $\hat{Q} \simeq S L_{2}\left(q_{1}\right), Z(\hat{Q}) \leqq$ $Z(\hat{L})$ and $\langle t, Y\rangle$ acts properly on $\hat{Q}$.

Then there exists $u \in \operatorname{Inv} \hat{L}$ such that $\hat{S}_{1}, \hat{S}_{2} \in \operatorname{Comp} C_{\hat{L}}(u)$ where $\widehat{S}_{1} \widehat{S}_{2} \simeq S L_{2}(q) \times S L_{2}(q), Z\left(\hat{S}_{1} \widehat{S}_{2}\right)=\langle u, Z(\hat{Q})\rangle$ and $\langle t, Y\rangle$ acts properly on both $\hat{S}_{1}$ and $\hat{S}_{2}$.

Proof. As before, we may suppose $L(X)=L(G(\rho))$ for suitable $G, \rho$. Let $\hat{G}$ denote the simply connected covering group of $G$ and lift the action of $\rho$ to $\hat{G}$, then $\hat{L} \subseteq \hat{G}(\rho)$. Since $|Z(\widehat{G})|$ must be even $G$ is not of type $A_{r}(r=$ even $)$ or $E_{6}$.

Consider all $v \in \operatorname{Inv}\left(\right.$ Aut $X$ ) with $Q \in \operatorname{Comp} C_{X}(v)$ such that $Q \simeq$ $L_{2}\left(q_{1}\right)$. Since $r \geqq 2$, by $\S 5.5$ in [3], $v$ cannot be a field-type auto- 
morphism. If $G$ is of type $A_{r}(r \geqq 5), C_{r}(r \geqq 3)$, or $E_{7}$ then the methods of $\S 4$ in [3] show that $v$ must be conjugate to $\hat{\alpha}_{*}(-1)$. Since $S_{*}=\left\langle U_{\alpha_{*}}, U_{-\alpha_{*}}\right\rangle$ is the unique simple rank 1 factor in $C_{G}\left(\hat{\alpha}_{*}(-1)\right)$ and $S_{*}(\rho) \simeq S L_{2}(q)$ we conclude that $G$ must be of type $B_{r}(r \geqq 2)$ or $D_{r}(r \geqq 3)$. For these cases we have, up to conjugacy in $G$, the following candidates for $s$ :

$$
\begin{aligned}
& B_{r}(r \geqq 2) \quad \underset{1}{\bigcirc}=\underset{2}{\bullet}-\underset{3}{\bullet}-\cdots--_{r}^{\bullet} s=\hat{\alpha}_{*}(-1) \text { or } \eta_{2}(-1) \\
& D_{r}(r \geqq 3) \underset{2 \bigcirc}{1 \bigcirc} \bigcirc-\cdots-\bigcirc_{r}^{\bigcirc} s=\hat{\alpha}^{*}(-1) \text { or } \eta_{r}(-1) \psi
\end{aligned}
$$

where $\psi$ is the standard form graph automorphism interchanging $\alpha_{1}$ and $\alpha_{2}$.

Put $S_{r}=\left\langle U_{\alpha_{r}}, U_{-\alpha_{r}}\right\rangle$ then $S_{r} \simeq S L_{2}(k)$ is a factor of $E\left(C_{G}\left(\hat{\alpha}_{*}(-1)\right)\right.$ and $S_{r} S_{*} \simeq S L_{2}(k) * S L_{2}(k)$ with $\left\langle\hat{\alpha}_{*}(-1)\right\rangle=Z\left(S_{r} S_{*}\right)$. As in the proof of Proposition A, we see that if $t=\eta_{i}(-1)$ then $\langle t, Y\rangle$ acts properly on both $S_{r}$ and $S_{*}$ except in one case, namely $t=\eta_{1}(-1)$ (or $\eta_{2}(-1)$ ) and $G$ of type $D_{r}$. However we can show that this case does not satisfy hypothesis (i) and (ii): Suppose $s=\eta_{r}(-1) \psi$, then a complete set of representatives for the classes of involutions in $C_{G}(s)^{\circ}$ are $\eta(-1)$ and $\left(\eta+\eta_{r}\right)(-1)$ in $\Gamma_{\psi}$ (see $\S 4.2$ in [3]) where $\eta \in\left\{\eta_{1}+\eta_{2}, \eta_{3}, \cdots, \eta_{r-1}\right\}$. Using the algorithm in Appendix 2 of [3] one shows that none of these involutions are conjugate in $G$ to either $\eta_{1}(-1)$ or $\eta_{2}(-1)$. For example $\left(\eta_{1}+\eta_{2}+\eta_{r}\right)(-1) \sim\left(\eta_{1}+\right.$ $\left.\eta_{2}-\eta_{r-1}+\eta_{r}\right)(-1) \sim\left(\eta_{1}+\eta_{2}-\eta_{r-2}+\eta_{r-1}\right)(-1) \sim \cdots \sim\left(\eta_{1}+\eta_{2}-\right.$ $\left.\eta_{3}+\eta_{4}\right)(-1) \sim \eta_{3}(-1)$ in $G$. Now classify the involutions in $C_{G}\left(\eta_{1}(-1)\right)$. Up to conjugacy in $C_{G}\left(\eta_{1}(-1)\right)$ we find that we may assume $s=\hat{\alpha}_{*}(-1)$. Hence if $\rho \eta_{1}(-1)=\eta_{1}(-1)$ and $s \in G(\rho), \rho$ must always stabilize both $S_{r}$ and $S_{*}$. Hence $\left(S_{r} S_{*}\right)(\rho) \simeq S L_{2}(q) * S L_{2}(q)$ (if $\rho$ flipped $S_{r}$ and $S_{*}$ then $\left(S_{r} S_{*}\right)(\rho) \simeq L_{2}\left(q^{2}\right) \times\langle s\rangle$ ) and so hypothesis (ii) is not satisfied.

Finally, note that we must have $\hat{L}=\hat{G}(\rho)$ since hypothesis (ii) is not satisfied for any intermediate covering when $G$ is of type $D_{r}$. Let $\hat{S}_{r}, \hat{S}_{*} \in \operatorname{Comp} C_{\widehat{\sigma}}(\gamma)$ where $u=\hat{\alpha}_{*}(-1)$. Then $\hat{S}_{r} \widehat{S}_{*} \simeq$ $S L_{2}(k) \times S L_{2}(k)$ and $Z\left(\widehat{S}_{r} \widehat{S}_{*}\right)=\langle u, Z(\hat{Q})\rangle . \quad$ As in the final step of the proof of Proposition A we can choose $\rho$ to stabilize $\eta_{1}(-1)$ and $\hat{S}_{r}$ and $\hat{S}_{*}$ and hence are done.

Proposition C. Let $X \in \mathscr{C}_{4}, r \in \operatorname{Inv} X, R_{1}, R_{2} \in \operatorname{Comp} C_{X}(r)$ such that $R_{1} \simeq L_{2}(q), R_{2} \simeq S L_{2}(q)$ and $\langle r\rangle=Z\left(R_{2}\right)$ then

(a) $L(X) \simeq B_{3}(q)$ and

(b) there is no $t \in \operatorname{Inv} X$ with $Y \subseteq O\left(C_{X}(t)\right) \neq 1$ such that $\langle t, Y\rangle$ acts properly on both $R_{1}$ and $R_{2}$. 
Proof. (a) follows from inspection of the centralizers of all elements in Inv (Aut $X),(L(X)=L(G(\rho))$, as before). For this, see the above table and related facts in [3].

So $G$ is of Type $B_{3}$. We make take $r=\hat{\alpha}_{*}(-1)$ and since $[t, r]=1$ can look for possible $t$ 's in $C_{G}(r)$. With $S_{j}=\left\langle U_{\alpha_{j}}, U_{-\alpha_{j}}\right\rangle$ we have $C_{G}(\delta)^{0}=S_{1} S_{3} S_{*}$ where $S_{1} \simeq L_{2}(k)$ and $S_{3} S_{*} \simeq S L_{2}(k) * S L_{2}(k)$. Elements in $C_{G}(r) / C_{G}(r)^{0}$ flip $S_{3}$ and $S_{*}$ and so $t \in C_{G}(r)^{0}$ (if it exists). There are 5 classes of involutions in $C_{G}(r)^{0}$ with representatives $\eta(-1)$ where $\eta \in\left\{\eta_{1}, \eta_{2}, \eta_{1}+\eta_{2}, \eta_{1}+\eta_{3}, \eta_{2}+\eta_{3}\right\}$. Only $\eta(-1)$ with $\eta \in\left\{\eta_{1}+\right.$ $\left.\eta_{2}, \eta_{2}+\eta_{2}\right\}$ are conjugate in $G$ to $\eta_{3}\left(-1\right.$ ) (in $B_{3}$ only $m_{3}=1$ ). Since $\alpha_{*}=-\left(2 \alpha_{1}+2 \alpha_{2}+\alpha_{3}\right)$ and since $Y \subseteq\left\langle\eta(\zeta): \zeta \in k^{*}\right\rangle$ we see that $\langle t, Y\rangle$ centralizes $S_{3}$ and $S_{*}$ if $\eta=\eta_{1}+\eta_{2}$ and $S_{1}$ if $\eta=\eta_{2}+\eta_{3}$. Hence there is no such $t$ and (b) is proved.

6. The $L_{6}(4)$ case. In Step 2 of the proof in $\S 4$ the case $L\left(J^{*}\right) \simeq L_{3}(4)$ may be eliminated as follows:

The involutions inside $L_{3}(4)$ have solvable, core-free, centralizers and hence both $\alpha, \beta$ induce outer automorphisms on $L\left(J^{*}\right)$. Put $\widehat{J}=J / O(J)$ then $L(\hat{J})$ is quasi-simple. Put $\hat{S}=S O(J) / O(J)$ then $\hat{S} \simeq S L_{2}(q)$ and $\hat{S} \in \operatorname{Comp} C_{\hat{J}}(\beta)$ (and $q=5$ or 7 ). This implies, by a direct calculation on $L_{3}(4)$, that $L(\hat{J})$ is the full 2-fold covering of $L_{3}(4)$ and $C_{L(\hat{\jmath})}(\beta)=\langle\hat{\rho}\rangle * \hat{S}$ where $\hat{\rho} \in Z(\hat{J}),\left\langle\hat{\rho}^{2}\right\rangle=Z(\hat{S})$ and $\hat{\rho}^{\alpha}=\hat{\rho}^{-1}$.

Since $Z(S)=\langle\gamma\rangle$ we may choose $\rho \in C_{J}(\beta)$, an inverse image of $\hat{\rho}$, satisfying $\rho^{2}=\gamma$. Then $\rho$ normalizes but does not centralize $M$ (see $\S 3$ ). Put $M_{1}=\langle M, \rho\rangle$ and $M_{1}^{*}=M_{1} / Z^{*}\left(M_{1}\right)$ and, for convenience, let $\alpha, \rho, \gamma$ also denote the images of these elements in $M_{1}^{*}$.

We may assume that $\alpha, \gamma$ are chosen as in Proposition $\mathrm{A}$ of $\S 5$. Since $\hat{\rho} \in Z(\hat{J})$ therefore $\rho$ centralizes every element of $\operatorname{Comp} C_{H_{1}^{*}}(\gamma)$. By the general structure of $C_{M_{1}}^{*}(\gamma)$ (see $\S \S 4$ and 5 in [3]) we must have $\rho \in\left\langle\hat{\alpha}_{*}(\zeta): \zeta \in k^{*}\right\rangle$ and hence $\rho$ and $\alpha=\eta_{i}(-1)$ commute. This contradicts the fact that $\hat{\rho}$ is inverted by $\alpha$.

The author wishes the thank M. Harris and R. Solomon for helpful comments and is also indebted to the referee for numerous useful suggestions.

\section{REFERENCES}

1. M. Aschbacher, Finite groups with a proper 2-generated core, Trans. Amer. Math. Soc., 197 (1975), 87-112.

2. - A characterization of Chevalley groups over fields of odd order, (preprint, 1976).

3. N. Burgoyne and C. Williamson, Semi-simple classes in Chevalley type groups, to appear in Pacific J. Math., 1977.

4. D. Goldschmidt, 2-signalizer functors on finite groups, J. Algebra, 21 (1972), 321-340.

5. D. Gorenstein and J. Walter, Balance and generation in finite groups, J. Algebra, 33 (1975), 224-287. 
6. D. Gorenstein and J. Walter, Centralizers of involutions in balanced groups, J. Algebra, 20 (1972), 284-319.

7. D. Gorenstein, Finite Groups, Harper and Row, N. Y., 1968.

8. D. Gorenstein and Harada, Finite groups whose 2-subgroups are generated by at most 4 elements, Mem. Amer. Math. Soc., No. 147 (1974).

9. D. Held, The simple groups related to $M_{24}$, J. Algebra, 13 (1969), 253-296.

10. N. IWAHORI, Centralizers of involutions in finite Chevalley groups, Springer Lect. Notes No. 131 (1970).

11. A. MacWilliams, On 2-groups with no normal abelian subgroups of rank 3, and their occurrence as Sylow 2-subgroups of finite simple groups, Trans. Amer. Math. Soc., 150 (1970), 345-408.

12. R. Solomon, Finite groups with intrinsic 2-components of type $\hat{A}_{n}$, J. Algebra, 33 (1975), 498-522.

13. J. Thompson, Notes on the B-conjecture (dittoed notes, 1974).

14. J. Walter, Characterization of Chevalley groups I, Proc. of Int. Symp. of Finite Groups, Sapporo 1974, 117-139.

Received July 1, 1976 and in revised form May 9, 1977. Work supported by NSF Grant MCS76-06555.

UnIVERSITY of CALIFoRNiA

SANTA Cruz, CA 95060 


\title{
PACIFIC JOURNAL OF MATHEMATICS
}

\section{EDITORS}

\author{
RICHARD ARENS (Managing Editor) \\ University of California \\ Los Angeles, CA 90024 \\ Charles W. Curtis \\ University of Oregon \\ Eugene, OR 97403 \\ C. C. MOORE \\ University of California \\ Berkeley, CA 94720
}

\section{J. DUGUNDJI}

Department of Mathematics

University of Southern California

Los Angeles, CA 90007

R. FINN and J. MILGRAM

Stanford University

Stanford, CA 94305

\section{ASSOCIATE EDITORS}
E. F. BECKENBACH
B. H. NeumanN
F. WOLF
K. YoSHIDA

\section{SUPPORTING INSTITUTIONS}

UNIVERSITY OF BRITISH COLUMBIA

CALIFORNIA INSTITUTE OF TECHNOLOGY

UNIVERSITY OF CALIFORNIA

MONTANA STATE UNIVERSITY

UNIVERSITY OF NEVADA, RENO

NEW MEXICO STATE UNIVERSITY

OREGON STATE UNIVERSITY

UNIVERSITY OF OREGON

OSAKA UNIVERSITY

\author{
UNIVERSITY OF SOUTHERN CALIFORNIA \\ STANFORD UNIVERSITY \\ UNIVERSITY OF HAWAII \\ UNIVERSITY OF TOKYO \\ UNIVERSITY OF UTAH \\ WASHINGTON STATE UNIVERSITY \\ UNIVERSITY OF WASHINGTON \\ $\stackrel{*}{*} \stackrel{*}{*} \stackrel{*}{*}$ AMERICAN MATHEMATICAL SOCIETY
}

The Supporting Institutions listed above contribute to the cost of publication of this Journal, but they are not owners or publishers and have no responsibility for its content or policies.

Mathematical papers intended for publication in the Pacific Jaurnal of Mathematics should be in typed form or offset-reproduced, (not dittoed), double spaced with large margins. Please do not use built up fractions in the text of your manuscript. You may however, use them in the displayed equations. Underline Greek letters in red, German in green, and script in blue. The first paragraph or two must be capable of being used separately as a synopsis of the entire paper. Items of the bibliography should not be cited there unless absolutely necessary, in which case they must be identified by author and Journal, rather than by item number. Manuscripts, in triplicate, may be sent to any one of the editors. Please classify according to the scheme of Math. Reviews, Index to Vol. 39. All other communications should be addressed to the managing editor, or Elaine Barth, University of California, Los Angeles, California, 90024.

The Pacific Journal of Mathematics expects the author's institution to pay page charges, and reserves the right to delay publication for nonpayment of charges in case of financial emergency.

100 reprints are provided free for each article, only if page charges have been substantially paid. Additional copies may be obtained at cost in multiples of 50 .

The Pacific Journal of Mathematics is issued monthly as of January 1966. Regular subscription rate: $\$ 7200$ a year (6 Vols., 12 issues). Special rate: $\$ 36.00$ a year to individual members of supporting institutions.

Subscriptions, orders for back numbers, and changes of address should be sent to Pacific Journal of Mathematics, 103 Highland Boulevard, Berkeley, California, 94708.

PUBLISHED BY PACIFIC JOURNAL OF MATHEMATICS, A NON-PROFIT CORPORATION

Printed at Kokusai Bunken Insatsusha (International Academic Printing Co., Ltd.). 8-8, 3-chome, Takadanobaba, Shinjuku-ku, Tokyo 160, Japan.

Copyright (C) 1975 by Pacific Journal of Mathematics Manufactured and first issued in Japan 


\section{Pacific Journal of Mathematics}

Vol. 72, No. 2

February, 1977

George E. Andrews, Plane partitions. II. The equivalence of the

Bender-Knuth and MacMahon conjectures ................. 283

Lee Wilson Badger, An Ehrenfeucht game for the multivariable quantifiers

of Malitz and some applications ......................... 293

Wayne C. Bell, A decomposition of additive set functions ............ 305

Bruce Blackadar, Infinite tensor products of $C^{*}$-algebras ............. 313

Arne Brøndsted, The inner aperture of a convex set .............. 335

N. Burgoyne, Finite groups with Chevalley-type components........... 341

Richard Dowell Byrd, Justin Thomas Lloyd and Roberto A. Mena, On the retractability of some one-relator groups .....................

Paul Robert Chernoff, Schrödinger and Dirac operators with singular potentials and hyperbolic equations .................... 361

John J. F. Fournier, Sharpness in Young's inequality for convolution ....... 383

Stanley Phillip Franklin and Barbara V. Smith Thomas, On the metrizability

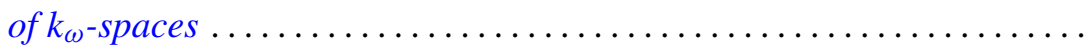

David Andrew Gay, Andrew McDaniel and William Yslas Vélez, Partially normal radical extensions of the rationals .................... 403

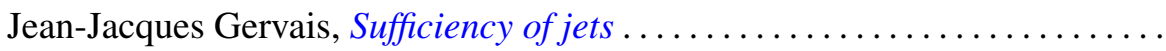

Kenneth R. Goodearl, Completions of regular rings. II . .

Sarah J. Gottlieb, Algebraic automorphisms of algebraic groups with stable maximal tori

Donald Gordon James, Invariant submodules of unimodular Hermitian forms.....

J. Kyle, $W_{\delta}(T)$ is convex.

Ernest A. Michael and Mary Ellen Rudin, A note on Eberlein compacts ...

Ernest A. Michael and Mary Ellen Rudin, Another note on Eberlein compacts ....

Thomas Bourque Muenzenberger and Raymond Earl Smithson, Fixed point theorems for acyclic and dendritic spaces.

Budh Singh Nashier and A. R. Rajwade, Determination of a unique solution of the quadratic partition for primes $p \equiv 1(\bmod 7)$.

Frederick J. Scott, New partial asymptotic stability results for nonlinear ordinary differential equations ....................

Frank Servedio, Affine open orbits, reductive isotropy groups, and dominant gradient morphisms; a theorem of Mikio Sato..........

D. Suryanarayana, On the distribution of some generalized square-full integers.................................. 